\title{
Reconstructing an Analytic Function Using Truncated Lagrange Polynomials
}

\author{
D. D. Trong and T. N. Lien
}

\begin{abstract}
Let $U$ be the unit disc of the complex plane. We consider the problem of reconstructing a function $f$ in the Hardy space $H^{2}(U)$ from values $f\left(z_{n}^{(m)}\right)$, where $\left\{z_{n}^{(m)}\right\} \quad(m \in \mathbb{N} ; 1 \leq n \leq m)$ is a given point system in $U$. This is an ill-posed problem. The function $f$ is approximated by so-called truncated Lagrange polynomials. Necessary and sufficient conditions for the convergence are established and a regularization result is given.

Keywords: Hardy spaces, truncated Lagrange polynomials, ill-posedness of problems, uniformly distributed point systems
\end{abstract}

AMS subject classification: 30D55, 30E05, 30E10, 65J20

\section{Introduction}

Let $U$ be the open unit disc of the complex plane $\mathbb{C}$ and let $H^{2}(U)$ be he Hardy space of all functions $f$, analytic in $U$ and satisfying

$$
\|f\|=\lim _{r \uparrow 1}\left\{\frac{1}{2 \pi} \int_{0}^{2 \pi}\left|f\left(r e^{i \theta}\right)\right|^{2} d \theta\right\}^{\frac{1}{2}}<\infty .
$$

We recall that, if $f \in H^{2}(U)$ has the expansion $f(z)=\sum_{k=0}^{\infty} \alpha_{k} z^{k}$, then $\|f\|=\left(\sum_{k=0}^{\infty}\left|\alpha_{k}\right|^{2}\right)^{1 / 2}$ (see, e.g., [10: Chapter 17]).

Let $\left\{z_{n}^{(m)}\right\} \quad(m \in \mathbb{N} ; 1 \leq n \leq m)$ be a point system in $U$. For each $m$, we assume that $z_{1}^{(m)}, \ldots, z_{m}^{(m)}$ are distinct points. In this paper, we consider the

Dang Duc Trong: Ho Chi Minh City Nat. Univ., Dept. Math. \& Comp. Sci., 227 Nguyen Van $\mathrm{Cu}$, Q5, Ho Chi Minh City, Vietnam ddtrong@mathdep.hcmuns.edu.vn Tran Ngoc Lien: Cantho Univ., Fac. Nat. Sci., Cantho City, Vietnam tnlien@ctu.edu.vn 
problem of reconstructing a function $f$ in $H^{2}(U)$ such that

$$
f\left(z_{n}^{(m)}\right)=\mu_{n}^{(m)} \quad(m \in \mathbb{N} ; 1 \leq n \leq m)
$$

where $\left\{\mu_{n}^{(m)}\right\}$ is a bounded set of complex numbers. Problem (1) has given rise to a huge literature. The reader is referred, e.g., to the monographs $[2$, $3,6,12]$ and the references therein. In fact, the unknown function $f$ is often approximated by polynomials (especially, by Lagrange polynomials, see, e.g., $[2,12])$ and by rational functions (see $[6,9,12]$ ).

On the other hand, problem (1) is ill-posed. In fact, the data $\mu_{n}^{(m)}$ in the right-hand side of (1) are given by measurements (or by rounding). Hence, the data are affected with errors. As a consequence, a solution of problem (1) corresponding to noise data does not always exist. Moreover, solutions, even when they do exist, do not depend continuously on the given data. Thus, the problem is intractable numerically and hence one has to resort to a regularization. In our recent results $[1,5,8,11]$ the ill-posedness of the problem was considered. We also refer to [6] in which a function $f$ in the disc algebra $A(U)$ is approximated by a sequence of functions constructed from noise data and called a robustly convergence identification algorithm.

The present paper deals with a regularization of problem (1) based on an approach of approximating (in $H^{2}(U)$ ) the function $f$ by polynomials

$$
L_{m}^{\theta}(\nu)(z)=\sum_{0 \leq k \leq \theta(m-1)} l_{k}^{(m)} z^{k} \quad\left(0<\theta \leq 1 ; \nu=\left(\mu_{1}^{(m)}, \ldots, \mu_{m}^{(m)}\right)\right) .
$$

Here $l_{k}^{(m)}$ is the coefficient of $z^{k}$ in the expansion of the Lagrange polynomial $L_{m}(\nu)$ of degree (at most ) $m-1$ satisfying

$$
L_{m}(\nu)\left(z_{k}^{(m)}\right)=\mu_{k}^{(m)} \quad(1 \leq k \leq m)
$$

The polynomial $L_{m}^{\theta}(\nu)$ is called a "truncated" Lagrange polynomial. We note that if $\theta=1$, then $L_{m}^{\theta}(\nu)$ is a Lagrange polynomial.

Before giving precise definitions and main results, several remarks are in order.

First, as is known, the convergence of $L_{m}(\nu)$ to $f$ depends heavily on properties of the point system $\left\{z_{n}^{(m)}\right\}$. The Kalmár-Walsh theorem (see, e.g., [2: Chapter 11]) shows that $L_{m}(\nu) \rightarrow f$ in $C(\bar{U})$ for all $f$ analytic in a neighborhood of $\bar{U}$ if and only if $\left\{z_{n}^{(m)}\right\}$ is uniformly distributed in $\bar{U}$, i.e.,

$$
\lim _{m \rightarrow \infty} \sqrt[m]{\max _{|z| \leq 1}\left|\omega_{m}(z)\right|}=1
$$


where

$$
\omega_{m}(z)=\left(z-z_{1}^{(m)}\right) \cdots\left(z-z_{m}^{(m)}\right) .
$$

This fact fails if $C(\bar{U})$ is replaced by $H^{2}(U)$ (see, e.g., Counterexample in Section 2). Moreover, condition (3) is very strict. In our paper, the point system $\left\{z_{n}^{(m)}\right\}$, in general, does not satisfy (3).

Note that the present approach, to our knowledge, is new. In $[1,5]$, truncated polynomials $L_{m}^{\frac{1}{2}}(\nu)$ are used to approximate the function $f$. In the present paper, we shall study the convergence of $L_{m}^{\theta}(\nu)$ for $\theta$ being in an open interval. In fact, we shall show that there is a $\theta_{0}$ in $(0,1)$ such that $L_{m}^{\theta}(\nu) \rightarrow f$ in $H^{2}(U)$ for $0<\theta<\theta_{0}$, and that the latter result does not hold if $\theta_{0}<\theta<1$.

Finally, if for each $m$ the nodes $z_{1}^{(m)}, \ldots, z_{m}^{(m)}$ are not mutually distinct and if at these points we know not only the values of $f$ but also the values of higher derivatives of $f$, then we can use the "truncated" Lagrange-Hermite polynomials to approximate the function $f$.

The remainder of our paper is divided into two sections. In Section 2, we shall state our main results and establish a counterexample. In Section 3, we shall prove the results stated in Section 2.

\section{Notations, main results and counterexample}

We put

$$
L_{m}(\nu)(z)=\sum_{n=1}^{m} \nu_{k} \frac{\omega_{m}(z)}{\omega_{m}^{\prime}\left(z_{k}^{(m)}\right)\left(z-z_{k}^{(m)}\right)}
$$

where $\omega_{m}$ is as in $(4), \nu=\left(\nu_{1}, \ldots, \nu_{m}\right)$ and put

$$
\begin{aligned}
\sigma_{0}^{(m)} & =1 \\
\sigma_{k, p}^{(m)} & =\sum_{1 \leq j_{1}<\ldots<j_{p} \leq m} z_{j_{1}}^{(m)} \cdots z_{j_{p}}^{(m)} \quad\left(j_{s} \neq k, 1 \leq s \leq p, p=1, \ldots, m-1\right) .
\end{aligned}
$$

We first state necessary conditions for the convergence of truncated Lagrange polynomials in $H^{2}(U)$.

Theorem 1. Let $0<\theta \leq 1$. If

$$
\lim _{m \rightarrow \infty}\left\|f-L_{m}^{\theta}\left(T_{m} f\right)(z)\right\|=0 \quad\left(f \in H^{2}(U)\right)
$$

where $L_{m}^{\theta}(\nu)$ is defined in $(2),(5)$ and

$$
T_{m}(f)=\left(f\left(z_{1}^{(m)}\right), \ldots, f\left(z_{m}^{(m)}\right)\right)
$$


then

$$
\sup _{m} \max _{1 \leq k \leq m} \prod_{j \in 1, m \backslash\{k\}}\left|1-\bar{z}_{j}^{(m)} z_{k}^{(m)}\right|^{-1}\left(\sum_{0 \leq \ell \leq \theta(m-1)}\left|\sigma_{k, m-1-\ell}^{(m)}\right|^{2}\right)^{\frac{1}{2}}<\infty
$$

where $\overline{1, m}=\{1, \ldots, m\}$.

Theorem 1 implies the following

Counterexample. We shall show that the Kalmár-Walsh theorem does not hold if $C(\bar{U})$ is replaced by $H^{2}(U)$. Indeed, putting $\theta=1$, for each $m$, $L_{m}^{\theta} T_{m} f$ is a Lagrange polynomial. Let $z_{n}^{(m)}=\frac{1}{n+1}(m \in \mathbb{N} ; 1 \leq n \leq m)$. We have

$$
\max _{|z| \leq 1}\left|\omega_{m}(z)\right|=\left(1+\frac{1}{2}\right) \cdots\left(1+\frac{1}{m+1}\right) .
$$

It follows that $\lim _{m \rightarrow \infty} \sqrt[m]{\max _{|z| \leq 1}\left|\omega_{m}(z)\right|}=1$, i.e. the system $\left\{z_{n}^{(m)}\right\}$ is uniformly distributed in $U$. On the other hand,

$$
\begin{aligned}
& \prod_{j \in 1, m \backslash\{m\}}\left|1-\bar{z}_{j}^{(m)} z_{m}^{(m)}\right|^{-1}\left(\sum_{0 \leq \ell \leq \theta(m-1)}\left|\sigma_{m, m-1-\ell}^{(m)}\right|^{2}\right)^{\frac{1}{2}} \\
& \quad \geq \prod_{j=1}^{m-1}\left|1-\frac{1}{(j+1)(m+1)}\right|^{-1}\left|\sigma_{m, 1}^{(m)}\right| \\
& \quad \geq \frac{1}{2}+\ldots+\frac{1}{m} \\
& \quad \rightarrow \infty
\end{aligned}
$$

as $m \rightarrow \infty$. Hence, using Theorem 1, we can find a function $f \in H^{2}(U)$ such that $L_{m} T_{m} f \nrightarrow f$ in $H^{2}(U)$ as $m \rightarrow \infty$.

To state sufficient conditions for the convergence of the truncated Lagrange polynomials, we shall consider point systems $\left\{z_{n}^{(m)}\right\}$ satisfying some properties. Letting $\sigma \in(0,1)$, we put

$$
A_{m}=\left\{n \in \mathbb{N} \mid 1 \leq n \leq m \text { and }\left|z_{n}^{(m)}\right| \geq \sigma\right\}
$$

We denote by $\mathcal{F}_{\sigma}$ the set of point systems $\left\{z_{n}^{(m)}\right\}$ in $U$ satisfying

$$
\begin{aligned}
\lim _{m \rightarrow \infty} \frac{\operatorname{card} A_{m}}{m} & =0 \\
\lim _{m \rightarrow \infty} \pi_{m} & =1
\end{aligned}
$$


where card $A_{m}$ is the number of elements of $A_{m}$ and

$$
\pi_{m}= \begin{cases}1 & \text { if } A_{m}=\emptyset \\ \left(\prod_{n \in A_{m}}\left(1-\left|z_{n}^{(m)}\right|\right)\right)^{\frac{1}{m}} & \text { if } A_{m} \neq \emptyset .\end{cases}
$$

Condition (7) means that almost of points of the system $\left\{z_{n}^{(m)}\right\}$ concentrate in the disc with radius $\sigma$ centered at 0 , and condition (8) means that $\left\{z_{n}^{(m)}\right\}$ is not too close to the boundary of the unit disc. Point systems $\left\{z_{n}^{(m)}\right\}$ in $\mathcal{F}_{\sigma}$, in general, do not satisfy condition (3).

For $0 \leq \theta \leq 1$ we put

$$
\phi(\theta)= \begin{cases}\frac{2 \sigma^{1-\theta}}{1-\sigma} & \text { if } \frac{1}{2}<\theta \leq 1 \\ \frac{\sigma^{1-\theta}}{(1-\sigma) \theta^{\theta}(1-\theta)^{1-\theta}} & \text { if } 0<\theta \leq \frac{1}{2} \\ \frac{\sigma}{1-\sigma} & \text { if } \theta=0 .\end{cases}
$$

We can verify that $\phi$ is continuous and strictly increasing in $[0,1]$. Moreover, $\phi(1)=2(1-\sigma)^{-1}>1$ and, for $\sigma \in\left(0, \frac{1}{2}\right), \phi(0)=\sigma(1-\sigma)^{-1}<1$. Hence, in this case, there exists uniquely a $\theta_{0} \in(0,1)$ such that

$$
\begin{aligned}
\phi\left(\theta_{0}\right) & =1 \\
\phi(\theta) & <1 \quad\left(0 \leq \theta<\theta_{0}\right) .
\end{aligned}
$$

Now, we have

Theorem 2. Let $\sigma \in(0,1)$ and $\theta \in(0,1]$. Let $L_{m}^{\theta}(\nu)$ be defined as in (2), (5) and let $T_{m}(f)$ be defined as in Theorem 1. Then:

(i) If $0<\sigma<\frac{1}{2}$, then

$$
\lim _{m \rightarrow \infty}\left\|f-L_{m}^{\theta}\left(T_{m} f\right)\right\|=0 \quad\left(f \in H^{2}(U)\right)
$$

for every point system $\left\{z_{n}^{(m)}\right\}$ in $\mathcal{F}_{\sigma}$ and every $0<\theta<\theta_{0}$. In addition, if $f^{\prime} \in H^{2}(U)$ and $\phi(\theta)<\delta<1$, then there exists an $m(\theta, \delta)$ such that

$$
\left\|f-L_{m}^{\theta}\left(T_{m} f\right)(z)\right\|^{2} \leq([(m-1) \theta]+1) \delta^{2 m}\|f\|^{2}+\frac{\left\|f^{\prime}\right\|^{2}}{(m-1)^{2} \theta^{2}}
$$

for $m>m(\theta, \delta)$ where $[x]$ is the greatest integer not exceeding $x$.

(ii) If $0<\sigma<\frac{1}{2}$, then we can find a point system $\left\{z_{n}^{(m)}\right\}$ in $\mathcal{F}_{\sigma}$ such that (10) fails for every $\theta_{0}<\theta<1$.

(iii) If $\frac{1}{2} \leq \sigma<1$, then we can find a point system $\left\{z_{n}^{(m)}\right\}$ in $\mathcal{F}_{\sigma}$ such that (10) fails for every $\theta \in(0,1)$. 
The theorem shows that $0<\sigma<\frac{1}{2}$ is a crucial condition for the convergence of truncated Lagrange polynomials.

Finally, to get the regularization result in the case of noisy data, we set some notations. Namely, we put

$$
D_{m}=\max _{1 \leq n \leq m}\left(\max _{|z| \leq 1}\left|\frac{\omega_{m}(z)}{\left(z-z_{n}^{(m)}\right) \omega_{m}^{\prime}\left(z_{n}^{(m)}\right)}\right|\right) .
$$

Let $\psi:[1, \infty) \rightarrow \mathbb{R}$ be an increasing function satisfying

$$
\begin{aligned}
\psi(m) & \geq m(m \theta+1) D_{m} \quad(m \geq 1) \\
\lim _{t \rightarrow \infty} \psi(t) & =+\infty
\end{aligned}
$$

Finally, put

$$
m(\varepsilon)=\left[\psi^{-1}\left(\varepsilon^{-\frac{1}{2}}\right)\right] .
$$

It is clear that $m(\varepsilon) \rightarrow \infty$ as $\varepsilon \downarrow 0$. Using the above notations, we have

Theorem 3. Let $\varepsilon>0,0<\sigma<\frac{1}{2}, 0<\theta<\theta_{0}$ and $\phi(\theta)<\delta<1$. Assume that $\mu=\left(\mu_{n}^{(m)}\right)$ satisfies

$$
\sup _{m}\left(\max _{1 \leq n \leq m}\left|f\left(z_{n}^{(m)}\right)-\mu_{n}^{(m)}\right|\right) \leq \varepsilon
$$

Then there is an $\varepsilon_{0}(\delta, \theta)$ such that, for every $0<\varepsilon<\varepsilon_{0}(\delta, \theta)$ and $f, f^{\prime} \in$ $H^{2}(U)$,

$$
\left\|f-L_{m(\varepsilon)}^{\theta}\left(T_{m(\varepsilon)}(\mu)\right)\right\| \leq \varepsilon^{\frac{1}{2}}+\delta^{m(\varepsilon)} \sqrt{m(\varepsilon) \theta+1}\|f\|+\frac{\left\|f^{\prime}\right\|}{(m(\varepsilon)-1) \theta}
$$

where $T_{m}(\mu)=\left(\mu_{1}^{(m)}, \ldots, \mu_{m}^{(m)}\right)$.

\section{Proofs}

In this section, we shall give the proof of Theorems 1 - 3 .

Proof of Theorem 1. If we consider the truncated Lagrange polynomials $L_{m}^{\theta}\left(T_{m} f\right)$ as the image of $f$ on $H^{2}(U)$, then $L_{m}^{\theta}\left(T_{m}\right) \quad(m \geq 1)$ can be seen as a sequence of linear operators on $H^{2}(U)$. We denote by $\left\|L_{m}^{\theta} T_{m}\right\|$ the norm of these operators. From the Banach-Steinhaus theorem, relation (6) implies $\sup _{m}\left\|L_{m}^{\theta} T_{m}\right\|<\infty$. Putting

$$
f_{m}(z)=\prod_{j \in \overline{1, m} \backslash\{k\}} \frac{z-z_{j}^{(m)}}{1-\bar{z}_{j}^{(m)} z}
$$


we have $\left\|f_{m}\right\|=1$. Hence, in view of the latter equality we get

$$
\left\|L_{m}^{\theta} T_{m} f_{m}\right\| \leq\left\|L_{m}^{\theta} T_{m}\right\|\left\|f_{m}\right\|=\left\|L_{m}^{\theta} T_{m}\right\|<\infty
$$

On the other hand, we have

$$
L_{m}\left(T_{m} f_{m}\right)(z)=\prod_{j \in \overline{1, m} \backslash\{k\}} \frac{z-z_{j}^{(m)}}{1-\bar{z}_{j}^{(m)} z_{k}^{(m)}} .
$$

By direct computation, one get

$$
L_{m}^{\theta}\left(T_{m} f_{m}\right)(z)=\frac{\sum_{0 \leq \ell \leq \theta(m-1)}(-1)^{m-1-\ell} \sigma_{k, m-1-\ell}^{(m)} z^{\ell}}{\prod_{j \in \overline{1, m} \backslash\{k\}}\left(1-\bar{z}_{j}^{(m)} z_{k}^{(m)}\right)} .
$$

It follows that

$$
\left\|L_{m}^{\theta}\left(T_{m} f_{m}\right)\right\|^{2}=\prod_{j \in \overline{1, m} \backslash\{k\}}\left|1-\bar{z}_{j}^{(m)} z_{k}^{(m)}\right|^{-2}\left(\sum_{0 \leq \ell \leq \theta(m-1)}\left|\sigma_{k, m-1-\ell}^{(m)}\right|^{2}\right) .
$$

Combining the latter equality with (14) completes the proof of Theorem 1

Proof of Theorem 2. We shall give the proof of the following three cases:

(i) $\frac{1}{2} \leq \sigma<1$

(ii) $0<\sigma<\frac{1}{2}$ and $\theta_{0}<\theta<1$

(iii) $0<\sigma<\frac{1}{2}$ and $0<\theta<\theta_{0}$.

(i) The Case $\frac{1}{2} \leq \sigma<1$. We construct a point system $\left\{z_{n}^{(m)}\right\}$ such that we can find a function $f \in H^{2}(U)$ satisfying $L^{\theta}\left(T_{m} f\right) \not f$ in $H^{2}(U)$ as $m \rightarrow \infty$ for every $\theta \in(0,1)$. For this let $z_{1}^{(m)}=1-\frac{1}{m}$ and $z_{m}^{(m)}=1-\frac{1}{2 m}$. Noting that, for $y_{s n}=\frac{1}{2}-\frac{1}{(m n+2)^{s}}$,

$$
\prod_{j=2}^{m-1}\left|\frac{y_{s j}}{1-\bar{y}_{s j} z_{m}^{(m)}}\right| \rightarrow\left(\frac{1}{1+\frac{1}{2 m}}\right)^{m-2} \quad(s \rightarrow \infty)
$$

we can choose $p_{m}$ such that

$$
\prod_{j=2}^{m-1}\left|\frac{y_{p_{m} j}}{1-\bar{y}_{p_{m} j} z_{m}^{(m)}}\right| \geq \frac{1}{2}\left(\frac{1}{1+\frac{1}{2 m}}\right)^{m-2}
$$


We put

$$
z_{n}^{(m)}=y_{p_{m} n}=\frac{1}{2}-\frac{1}{(m n+2)^{p_{m}}} \quad(m \in \mathbb{N} ; 2 \leq n \leq m-1) .
$$

For $m \geq 3$, we have $A_{m}=\{1, m\} \quad\left(\operatorname{card} A_{m}=2\right)$. Hence, the point system $\left\{z_{n}^{(m)}\right\}$ satisfies $(7)-(8)$, i.e. $\left(z_{n}^{(m)}\right) \in \mathcal{F}_{\sigma}$. On the other hand, one has

$$
\begin{aligned}
& \prod_{j \in \frac{1, m}{1, m}\{m\}}\left|1-\bar{z}_{j}^{(m)} z_{m}^{(m)}\right|^{-1}\left(\sum_{0 \leq \ell \leq \theta(m-1)}\left|\sigma_{m, m-1-\ell}^{(m)}\right|^{2}\right)^{\frac{1}{2}} \\
& \geq\left|\sigma_{m, m-1}^{(m)}\right| \prod_{j=1}^{m-1}\left|1-\bar{z}_{j}^{(m)} z_{m}^{(m)}\right|^{-1} \\
& =\frac{\left|z_{1}^{(m)}\right|}{\left|1-\bar{z}_{1}^{(m)} z_{m}^{(m)}\right|} \prod_{j=2}^{m-1}\left|\frac{z_{j}^{(m)}}{1-\bar{z}_{j}^{(m)} z_{m}^{(m)}}\right| \\
& \geq \frac{\left|z_{1}^{(m)}\right|}{\left|1-\bar{z}_{1}^{(m)} z_{m}^{(m)}\right|} \frac{1}{2}\left(\frac{1}{1+\frac{1}{2 m}}\right)^{m-2} \\
& \rightarrow \infty
\end{aligned}
$$

as $m \rightarrow \infty$. Hence, from Theorem 1, (10) fails as desired.

(ii) The Case $0<\sigma<\frac{1}{2}$ and $\theta_{0}<\theta<1$. We construct a point system $\left\{z_{n}^{(m)}\right\}$ such that there exists $f \in H^{2}(U)$ satisfying $L_{m}^{\theta} T_{m} f \nrightarrow f$ in $H^{2}(U)$ for every $\theta_{0}<\theta<1$. In fact, we shall argue as in the foregoing case. For any $\sigma \in\left(0, \frac{1}{2}\right)$, let $z_{n}^{(m)}=\sigma\left(1-\frac{1}{m^{3}+n}\right)$ and $z_{m}^{(m)}=1-\frac{1}{m^{2}}$ for $n=1, \ldots, m-1$. We have $A_{m}=\{m\} \quad\left(\operatorname{card} A_{m}=1\right)$ for every $m \geq 2$. Hence, the point system $\left\{z_{n}^{(m)}\right\}$ satisfies $(7)-(8)$, i.e. it is in $\mathcal{F}_{\sigma}$. One has

$$
\begin{aligned}
& \prod_{j \in 1, m \backslash\{m\}}\left|1-\bar{z}_{j}^{(m)} z_{m}^{(m)}\right|^{-1}\left(\sum_{0 \leq \ell \leq \theta(m-1)}\left|\sigma_{m, m-1-\ell}^{(m)}\right|^{2}\right)^{\frac{1}{2}}\left|1-\bar{z}_{j}^{(m)} z_{m}^{(m)}\right|^{-1}\left|\sigma_{m, m-[m \theta]}^{(m)}\right| \\
& \geq\left.\prod_{j \in 1, m \backslash\{m\}} \sum \sum_{j=1}\left|\prod_{1 \leq j_{1}<\ldots<j_{m-[m \theta]} \leq m-1} \frac{1}{\left|1-z_{j}^{(m)} z_{m}^{(m)}\right|} z_{j_{1}}^{(m)} \cdots z_{j_{m-[m \theta]}}^{(m)}\right|\right|_{m-[m \theta]} C_{m}^{[m \theta]} \\
& \geq \frac{1}{\left[1-\sigma\left(1-\frac{1}{m^{3}+m}\right)\left(1-\frac{1}{m^{2}}\right)\right]^{m-1}} \sigma^{m-[m \theta]}\left(1-\frac{1}{m^{3}+1}\right)^{m-1}
\end{aligned}
$$


where $C_{m}^{k}=\frac{m !}{k !(m-k) !}$. Using Stirling's formula (see, e.g., [7]) we get

$$
\begin{aligned}
\lim _{m \rightarrow \infty} \sqrt[m]{C_{m}^{[m \theta]}} & =\lim _{m \rightarrow \infty}\left(\frac{m !}{[m \theta] !(m-[m \theta]) !}\right)^{\frac{1}{m}} \\
& =\lim _{m \rightarrow \infty} \frac{\frac{m}{e}}{\left(\frac{[m \theta]}{e}\right)^{\frac{[m \theta]}{m}}\left(\frac{m-[m \theta]}{e}\right)^{\frac{m-[m \theta]}{m}}} \\
& =\frac{1}{\theta^{\theta}(1-\theta)^{1-\theta}} .
\end{aligned}
$$

It follows that

$$
\begin{aligned}
& \limsup _{m \rightarrow \infty}\left(\prod_{j \in 1, m \backslash\{m\}}\left|1-\bar{z}_{j}^{(m)} z_{m}^{(m)}\right|^{-1}\left(\sum_{0 \leq \ell \leq \theta(m-1)}\left|\sigma_{m, m-1-\ell}^{(m)}\right|^{2}\right)^{\frac{1}{2}}\right)^{\frac{1}{m}} \\
& \quad \geq \frac{\sigma^{1-\theta}}{(1-\sigma) \theta^{\theta}(1-\theta)^{1-\theta}} \\
& \quad=\phi(\theta) \\
& >\phi\left(\theta_{0}\right) \\
& \quad=1 .
\end{aligned}
$$

Hence,

$$
\limsup _{m \rightarrow \infty} \prod_{j \in \overline{1, m} \backslash\{m\}}\left|1-\bar{z}_{j}^{(m)} z_{m}^{(m)}\right|^{-1}\left(\sum_{0 \leq \ell \leq \theta(m-1)}\left|\sigma_{m, m-1-\ell}^{(m)}\right|^{2}\right)^{\frac{1}{2}}=\infty .
$$

Using Theorem 1, we can find an $f \in H^{2}(U)$ such that $L_{m}^{\theta} T_{m} f \not f$ in $H^{2}(U)$.

(iii) The Case $0<\sigma<\frac{1}{2}$ and $0<\theta<\theta_{0}$. Assume that

$$
f(z)=\sum_{k=0}^{\infty} \alpha_{k} z^{k}, \quad \sum_{k=0}^{\infty}\left|\alpha_{k}\right|^{2}<\infty
$$

Since $L_{m}\left(T_{m} f\right)$ is a polynomial having degree $\operatorname{deg} L_{m}\left(T_{m} f\right) \leq m-1$, we can write

$$
L_{m}\left(T_{m} f\right)(z)=\sum_{k=0}^{m-1} l_{k}^{(m)} z^{k}
$$

where $l_{k}^{(m)}$ are constants. Substracting (17) from (16) gives

$$
f(z)-L_{m}\left(T_{m} f\right)(z)=\sum_{k=0}^{m-1} \delta_{k}^{(m)} z^{k}+\sum_{k=m}^{\infty} \alpha_{k} z^{k}
$$


where $\delta_{k}^{(m)}=\alpha_{k}-l_{k}^{(m)} \quad(1 \leq k \leq m)$.

On the other hand, the Hermite representation (cf. [2]) gives

$$
f(z)-L_{m}\left(T_{m} f\right)(z)=\frac{1}{2 \pi i} \int_{\partial U} \frac{\omega_{m}(z) f(\zeta) d \zeta}{\omega_{m}(\zeta)(\zeta-z)} .
$$

Direct computation gives

$$
\begin{aligned}
\omega_{m}(z) & =\sum_{r=0}^{m}(-1)^{m-r} \sigma_{m-r}^{(m)} z^{r} \\
\frac{1}{2 \pi i} \int_{\partial U} \frac{f(\zeta) d \zeta}{\omega_{m}(\zeta)(\zeta-z)} & =\sum_{s=0}^{\infty} \beta_{s}^{(m)} z^{s}
\end{aligned}
$$

where

$$
\begin{aligned}
\sigma_{0}^{(m)} & =1 \\
\sigma_{r}^{(m)} & =\sum_{1 \leq j_{1}<\ldots<j_{r} \leq m} z_{j_{1}}^{(m)} \cdots z_{j_{r}}^{(m)} \quad(1 \leq r \leq m) \\
\beta_{s}^{(m)} & =\frac{1}{2 \pi i} \int_{\partial U} \frac{f(\zeta) d \zeta}{\zeta^{s+1} \omega_{m}(\zeta)} \quad(s \geq 0) .
\end{aligned}
$$

Multiplying (20) and (21) together and substituting the result thus obtained into (19) we get

$$
f(z)-L_{m}\left(T_{m} f\right)(z)=\sum_{k=0}^{\infty}\left(\sum_{r=0}^{k}(-1)^{m-r} \sigma_{m-r}^{(m)} \beta_{k-r}^{(m)}\right) z^{k}
$$

where we put $\sigma_{-1}=\sigma_{-2}=\ldots=0$. From (18) and (23) it follows that

$$
\delta_{k}^{(m)}=\sum_{r=0}^{k}(-1)^{m-r} \sigma_{m-r}^{(m)} \beta_{k-r}^{(m)} \quad(0 \leq k \leq m-1) .
$$

We shall estimate $\delta_{k}^{(m)}$. We first have

$$
\left|\beta_{s}^{(m)}\right|=\left|\frac{1}{2 \pi i} \int_{\partial U} \frac{f(\zeta) d \zeta}{\zeta^{s+1} \omega_{m}(\zeta)}\right| \leq \frac{1}{\sqrt{2 \pi}}\|f\|\left(\int_{0}^{2 \pi} \frac{d \varphi}{\left|\omega_{m}\left(e^{i \varphi}\right)\right|^{2}}\right)^{\frac{1}{2}}
$$

On the other hand, $\left|\omega_{m}(z)\right| \geq \prod_{j=1}^{m}\left(1-\left|z_{j}^{(m)}\right|\right)$. Hence

$$
\left|\beta_{s}^{(m)}\right| \leq \frac{\|f\|}{\prod_{j=1}^{m}\left(1-\left|z_{j}^{(m)}\right|\right)} \leq \frac{\|f\|}{(1-\sigma)^{m-\operatorname{card} A_{m}} \prod_{j \in A_{m}}\left(1-\left|z_{j}^{(m)}\right|\right)}
$$


From (22), one has

$$
\begin{aligned}
\left|\sigma_{m-k}^{(m)}\right| & \leq \sum_{1 \leq j_{1}<\ldots<j_{m-k} \leq m}\left|z_{j_{1}}^{(m)} \cdots z_{j_{m-k}}^{(m)}\right| \\
& \leq \sigma^{m-k-\operatorname{card} A_{m}} \sum_{1 \leq j_{1}<\ldots<j_{m-k} \leq m} 1 \\
& =\sigma^{m-k-\operatorname{card} A_{m}} C_{m}^{k} .
\end{aligned}
$$

It follows that, for $0 \leq k \leq[m \theta]$,

$$
\left|\sigma_{m-k}^{(m)}\right| \leq \sigma^{m-[m \theta]-\operatorname{card} A_{m}} C_{m}^{k} .
$$

We have the following lemma (which will be proved later):

Lemma 1. Under the assumptions of Theorem 2,

$$
\limsup _{m \rightarrow \infty}\left(\max _{0 \leq k \leq[m \theta]}\left|\sigma_{m-k}^{(m)}\right|^{\frac{1}{m}}\right) \leq(1-\sigma) \phi(\theta)
$$

for $0<\theta<1$.

From (24), one has

$$
\left|\delta_{k}^{(m)}\right| \leq \frac{(1+m \theta)\|f\|}{(1-\sigma)^{m-\operatorname{card} A_{m}} \prod_{j \in A_{m}}\left(1-\left|z_{j}^{(m)}\right|\right)} \max _{0 \leq k \leq[m \theta]}\left|\sigma_{m-k}^{(m)}\right|
$$

for $0 \leq k \leq[m \theta]$. Combining the latter inequality with (27) and (8) gives

$$
\limsup _{m \rightarrow \infty}\left(\max _{0 \leq k \leq[m \theta]}\left|\frac{\delta_{k}^{(m)}}{\|f\|}\right|^{\frac{1}{m}}\right) \leq \phi(\theta) .
$$

Now, for $0<\theta<\theta_{0}$, in view of (9) and (28) we get

$$
\limsup _{m \rightarrow \infty}\left(\max _{0 \leq k \leq[m \theta]}\left|\frac{\delta_{k}^{(m)}}{\|f\|}\right|^{\frac{1}{m}}\right) \leq \phi(\theta)<1 .
$$

Hence, for $\phi(\theta)<\delta<1$ we can find an $m(\delta)>0$ such that

$$
\max _{0 \leq k \leq[m \theta]}\left|\delta_{k}^{(m)}\right|<\delta^{m}\|f\| \quad(m>m(\delta)) .
$$

From (16) - (17) one has

$$
f(z)-L_{m}^{\theta} T_{m} f(z)=\sum_{k=0}^{[(m-1) \theta]} \delta_{k}^{(m)} z^{k}+\sum_{k=[(m-1) \theta]+1}^{\infty} \alpha_{k} z^{k} .
$$


It follows that

$$
\left\|f-L_{m}^{\theta} T_{m} f\right\|^{2}=\sum_{k=0}^{[(m-1) \theta]}\left|\delta_{k}^{(m)}\right|^{2}+\sum_{k=[(m-1) \theta]+1}^{\infty}\left|\alpha_{k}\right|^{2} .
$$

Hence, from (30) we get

$$
\left\|f-L_{m}^{\theta} T_{m} f\right\|^{2} \leq([(m-1) \theta]+1) \delta^{2 m}\|f\|^{2}+\sum_{k=[(m-1) \theta]+1}^{\infty}\left|\alpha_{k}\right|^{2} .
$$

It follows that $L_{m}^{\theta} T_{m} f \rightarrow f$ in $H^{2}(U)$ as $m \rightarrow \infty$. If $f^{\prime} \in H^{2}(U)$, then we get $f^{\prime}(z)=\sum_{k=0}^{\infty} k \alpha_{k} z^{k-1}$ and $\left\|f^{\prime}\right\|=\left(\sum_{k=0}^{\infty} k^{2}\left|\alpha_{k}\right|^{2}\right)^{1 / 2}$. This gives

$$
\sum_{k=[(m-1) \theta]+1}^{\infty}\left|\alpha_{k}\right|^{2} \leq \frac{\left\|f^{\prime}\right\|^{2}}{(m-1)^{2} \theta^{2}}
$$

Combining (31) and (32) we get

$$
\left\|f-L_{m}^{\theta} T_{m} f\right\|^{2} \leq([(m-1) \theta]-1) \delta^{2 m}\|f\|^{2}+\frac{\left\|f^{\prime}\right\|^{2}}{(m-1)^{2} \theta^{2}} .
$$

This will complete the proof of Theorem 2 once Lemma 1 is proved

Proof of Lemma 1. We have to consider the cases

(i) $0<\theta<\frac{1}{2}$

(ii) $\frac{1}{2} \leq \theta<1$.

If (i) holds, then $C_{m}^{1} \leq \ldots \leq C_{m}^{[m \theta]}$. Hence (26) implies

$$
\max _{0 \leq k \leq[m \theta]}\left|\sigma_{m-k}^{(m)}\right|^{\frac{1}{m}} \leq \sigma^{1-\frac{[m \theta]+\operatorname{card} A_{m}}{m}} \sqrt[m]{C_{m}^{[m \theta]}} .
$$

From $(33),(15)$ and the assumption $\lim _{m \rightarrow \infty} \frac{\operatorname{card} A_{m}}{m}=0$ we have

$$
\limsup _{m \rightarrow \infty}\left(\max _{0 \leq k \leq[m \theta]}\left|\sigma_{m-k}^{(m)}\right|^{\frac{1}{m}}\right) \leq \frac{\sigma^{1-\theta}}{\theta^{\theta}(1-\theta)^{1-\theta}}=(1-\sigma) \phi(\theta) .
$$

If (ii) holds, from (26) we get

$$
\max _{0 \leq k \leq[m \theta]}\left|\sigma_{m-k}^{(m)}\right|^{\frac{1}{m}} \leq 2 \sigma^{1-\frac{[m \theta]+\operatorname{card} A_{m}}{m}}
$$

where we have used the identity $\sum_{k=0}^{m} C_{m}^{k}=2^{m}$. From here it follows that

$$
\limsup _{m \rightarrow \infty}\left(\max _{0 \leq k \leq[m \theta]}\left|\sigma_{m-k}^{(m)}\right|^{\frac{1}{m}}\right) \leq 2 \sigma^{1-\theta}=(1-\sigma) \phi(\theta) .
$$

Hence, in either case, inequality (27) holds. This completes the proof of Lemma 1 and the proof of Theorem 2 
Proof of Theorem 3. We first prove that

$$
\left\|L_{m}^{\theta}\left(T_{m} f\right)-L_{m}^{\theta}\left(T_{\mu}\right)\right\|_{\infty} \leq \varepsilon m(1+m \theta) D_{m} .
$$

In fact, we have

$$
\left(L_{m}\left(T_{m} f\right)-L_{m}\left(T_{\mu}\right)\right)(z)=\sum_{n=0}^{m}\left(f\left(z_{n}^{(m)}\right)-\mu_{n}^{(m)}\right) \frac{\omega_{m}(z)}{\left(z-z_{n}^{(m)}\right) \omega_{m}^{\prime}\left(z_{n}^{(m)}\right)} .
$$

By direct computation, we get

$$
\left|\left(L_{m}\left(T_{m} f\right)-L_{m}\left(T_{\mu}\right)\right)(z)\right| \leq \varepsilon m D_{m} \quad(|z| \leq 1) .
$$

On the other hand, one has

$$
\left(L_{m}\left(T_{m} f\right)-L_{m}\left(T_{\mu}\right)\right)(z)=\sum_{k=0}^{m-1} \lambda_{k}^{(m)} z^{k}
$$

where

$$
\lambda_{k}^{(m)}=\frac{1}{2 \pi i} \int_{\partial U} \frac{\left(L_{m}\left(T_{m} f\right)-L_{m}\left(T_{\mu}\right)\right)(\zeta) d \zeta}{\zeta^{k+1}} .
$$

From (36) and (38) one has

$$
\left|\lambda_{k}^{(m)}\right| \leq \varepsilon m D_{m}
$$

It follows from (37) and (39) that

$$
\left|L_{m}^{\theta}\left(T_{m} f\right)(z)-L_{m}^{\theta}\left(T_{\mu}\right)(z)\right|=\left|\sum_{k \leq[(m-1) \theta]} \lambda_{k}^{(m)} z^{k}\right| \leq \varepsilon m(m \theta+1) D_{m},
$$

i.e. (35) holds.

Now, we have

$$
\begin{aligned}
\| f & -L_{m(\varepsilon)}^{\theta}\left(T_{m(\varepsilon)}(\mu)\right) \| \\
& \leq\left\|f-L_{m(\varepsilon)}^{\theta}\left(T_{m(\varepsilon)} f\right)\right\|+\left\|L_{m(\varepsilon)}^{\theta}\left(T_{m(\varepsilon)}(\mu)\right)-L_{m(\varepsilon)}^{\theta}\left(T_{m(\varepsilon)} f\right)\right\| .
\end{aligned}
$$

Thus, using Theorem 2 and (35) we get

$$
\begin{aligned}
\| f & -L_{m(\varepsilon)}^{\theta}\left(T_{m(\varepsilon)}(\mu)\right) \| \\
& \leq \sqrt{m(\varepsilon) \theta+1} \delta^{m(\varepsilon)}\|f\|+\frac{\left\|f^{\prime}\right\|}{(m(\varepsilon)-1) \theta}+\varepsilon m(\varepsilon)(m(\varepsilon) \theta+1) D_{m(\varepsilon)} .
\end{aligned}
$$

From the definition of $m(\varepsilon)$ we have

$$
\varepsilon^{-\frac{1}{2}} \geq \psi(m(\varepsilon)) \geq m(\varepsilon)(m(\varepsilon) \theta+1) D_{m(\varepsilon)} .
$$

Hence,

$$
\left\|f-L_{m(\varepsilon)}^{\theta}\left(T_{m(\varepsilon)}(\mu)\right)\right\| \leq \varepsilon^{\frac{1}{2}}+\sqrt{m(\varepsilon) \theta+1} \delta^{m(\varepsilon)}\|f\|+\frac{\left\|f^{\prime}\right\|}{(m(\varepsilon)-1) \theta}
$$

which completes the proof of Theorem 3 
Acknowledgments. We would like to thank the reviewers for their frank, constructive criticism, leading to the present improvent of our paper. The first author would like to thank Dr. J. P. Calvi for his valuable discussion related to the Kalmár-Walsh theorem when the author visited Lab. Emile Picard, Toulouse, France in the spring 2000.

\section{References}

[1] Ang, D. D., Gorenflo, R., Le, V. K. and D. D. Trong: Moment Theory and Some Inverse Problems in Potential Theory and Heat Conduction. Lect. Notes Math. 1792 (2002).

[2] Gaier, D.: Vorlesungen über Approximation im Komplexen. Basel - Boston Stutgart: Birkhauser Verlag 1980.

[3] Guelfond, A. O.: Calcul des Différences Finis. Paris: Dunod 1963.

[4] Hoffman, K.: Banach Spaces of Analytic Functions. Englewood Cliffs (N.J., USA): Prentice-Hall Inc. 1962.

[5] Huy, N. V., Nhan, N. V. and D. D. Trong: Reconstruction of Analytic Functions on the Unit Disc from a Sequence of Moments: Regularization and Error Estimates. Acta Math. Vietnamica 27 (2002), 307 - 320.

[6] Partington, J. R.: Interpolation, Identification, and Sampling. Oxford: Clarendon Press 1997.

[7] Taylor, A.: Advanced Calculus. New York et al.: Blaisdell Publ. Comp. 1965.

[8] Trong, D. D., Nam, L. Q., Luc, N. L. and T. T. Tuyen: Reconstruction of $H^{p}$-Functions: Best Approximation, Regularization and Optimal Error Estimates. J. Math. Anal. Appl. (submitted 2002).

[9] Totik, V.: Recovery of $H^{p}-$ Functions. Proc. Amer. Math. Soc. 90 (1984), $531-537$.

[10] Rudin, W.: Real and Complex Analysis. New York et al.: McGraw-Hill Co. 1987.

[11] Trong, D. D. and D. D. Ang: Reconstruction of Analytic Functions: Regularization and Optimal Recovery. Preprint 1997.

[12] Walsh, J. L.: Interpolation and Approximation by Rational Functions in the Complex Domain. Providence (R.I., USA): Amer. Math. Soc. 1960.

Received 24.03.2003; in revised form 21.10.2003 